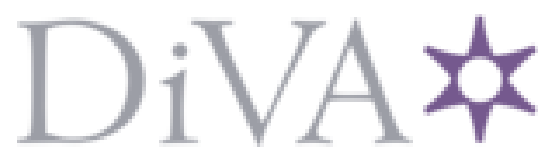

http://www.diva-portal.org

\title{
Postprint
}

This is the accepted version of a paper presented at The 2nd International Conference on Connected Vehicles \& Expo (ICCVE), Las Vegas, Dec 2-6, 2013.

Citation for the original published paper:

Vernersson, S., Kalpaxidou, E., Rylander, D. (2013)

Evaluation of Wireless Short-Range Communication Performance in a Quarry Environment. In: 2013 International Conference on Connected Vehicles and Expo (ICCVE) (pp. 308-313). http://dx.doi.org/10.1109/ICCVE.2013.6799812

N.B. When citing this work, cite the original published paper.

(c) 2014 IEEE. Personal use of this material is permitted. Permission from IEEE must be obtained for all other users, including reprinting/ republishing this material for advertising or promotional purposes, creating new collective works for resale or redistribution to servers or lists, or reuse of any copyrighted components of this work in other works.

Permanent link to this version:

http://urn.kb.se/resolve?urn=urn:nbn:se:mdh:diva-2631 1 


\section{Evaluation of Wireless Short-Range Communication Performance in a Quarry Environment}

\author{
Susanne Vernersson \\ Chalmers University of Technology \\ Gothenburg, Sweden \\ susanne.vernersson@gmail.com
}

\author{
Eleni Kalpaxidou \\ Chalmers University of Technology \\ Gothenburg, Sweden \\ eleni.kalpaxidou@gmail.com
}

\author{
David Rylander \\ Volvo Construction Equipment \\ Gothenburg, Sweden \\ david.rylander@volvo.com
}

\begin{abstract}
The quarry industry provides sand and gravel to produce the aggregates used to construct buildings and road structures. Productivity and safety within this industry can be improved by using wireless communication technologies. EMC, dust and solid materials that present non-line-of-sight (NLOS) issues create a harsh environment that poses challenges to using wireless communication. This paper evaluates how a set of wireless standards performs in the quarry in terms of range and packet reception ratio (PRR). The assessment includes the wireless short-range technologies ZigBee, 802.11g and 802.11p using frequencies of $868 \mathrm{MHz}, 2.4 \mathrm{GHz}$ and $5.9 \mathrm{GHz}$. We present measurement results from a real quarry environment and identify system considerations for quarry safety and efficiency applications based on collected data.
\end{abstract}

Keywords-Vehicular Communication, VANET, V2V/V2I, Wireless Communication, Quarry, DSRC, 802.11p, 802.11g, ZigBee

\section{INTRODUCTION}

Vehicle-to-vehicle (V2V) and vehicle-to-infrastructure (V2I) communication is increasingly being used to improve road safety and traffic efficiency. The proposed standard in Europe and the U.S. has so far been IEEE $802.11 \mathrm{p}$ at $5.9 \mathrm{GHz}$ via a vehicular ad hoc network (VANET) [1]. To date, few investigations address the possibilities of bringing intelligent transport systems (ITS) to an important industry - quarries. In Europe alone, there are over 24000 quarries with an annual demand of three billion tons, which translates to a 20 billion euro turnover [2]. The quarry industry is thus a promising, relatively unexplored market for wireless communication.

The road and quarry environments present some interesting differences for VANET technologies. Besides the obvious lack of road maps for quarries, the quarries normally have less vehicle variation and turnover. Most of the vehicles within a quarry remain at the site for their entire lifecycle. Furthermore, the quarry layout changes frequently over time due to the work that takes place at the site. For these reasons, the communication solutions for a quarry can be chosen without the same consideration to interoperability as for road vehicles. It may therefore be sufficient to be able to just communicate between vehicles working at the site and not with the general road vehicles.

Using wireless communication in quarries can increase safety and optimize productivity. For instance, a productivity increase of up to $30 \%$ [3] can be achieved assuming reliable wireless connectivity to minimize waste in production. In addition, $41 \%$ of all accidents in quarries are vehicle-related, with common incidents being "run over by a vehicle, trapped under vehicle body, vehicles colliding with plant or other vehicles, vehicle overturned on quarry floor or road and vehicles running over open edge of quarry face bench or ramp" [4]. This highlights a potential for accident avoidance using wireless communication-based warning systems.

Increasing safety and fuel efficiency enhancements in quarries using wireless communication reveals some interesting challenges for which non-functional requirements can be identified. Since quarries often are remotely located, a global solution requires instant coverage within a quarry pit. It cannot be assumed that a vehicle leaves a pit for better coverage to exchange data. This requirement excludes a cellular communication solution, since coverage cannot be guaranteed. Additionally, since safety applications require low latency communication to be reliable and trustworthy, satellitebased communication must also be excluded. Instead, a dedicated short-range communication-based (DSRC) solution is required for the stated purposes and needs.

Nevertheless, the conditions in a quarry are harsh for shortrange communication. There are lots of solid materials, and the terrain is often hilly, with plenty of obstacles leading to nonline-of-sight issues. High EMC and dust that may affect communication performance can also be expected. It is thus imperative to select a wireless standard for the quarry that can provide robust communication despite the challenging conditions. In Europe, there are three main, open, license-free bands available for wireless short-range communication. These are found at $868 \mathrm{MHz}, 2.4 \mathrm{GHz}$, and $5.9 \mathrm{GHz}$. Several standard protocols are available from suppliers, including many of the IEEE 802.11 amendments. Here we limit our evaluation to investigate ZigBee, 802.11g and 802.11p.

ZigBee is a standard that defines a set of communication protocols for reliable, cost-effective, low-data, short-range networking. It operates at $868 \mathrm{MHz}$ (in the E.U.), $902-928 \mathrm{MHz}$ (in the U.S.) and $2.4 \mathrm{GHz}$ (worldwide) [5]. ZigBee is often used in home automation, consumer electronics, industrial controls, and games [6]. The radio frequency (RF) protocol at $868 \mathrm{MHz}$ is reported to reach distances of up to $12 \mathrm{~km}$ in line-of-sight (LOS) [7].

IEEE $802.11 \mathrm{~g}$ extends the $802.11 \mathrm{~b}$ amendment to data rates from 12 up to $54 \mathrm{Mbps}$. It operates in the $2.4 \mathrm{GHz}$ band, and - 
just like its predecessor - 802.11g suffers from interference from products operating in the same frequency band [8]. The protocol is usually found in routers for home and office use, although recent routers tend to support the latest $802.11 \mathrm{n}$ amendment. IEEE reports the maximum measured outdoor range for $802.11 \mathrm{~b}$ and $802.11 \mathrm{~g}$ to be about 152 meters [9].

Another amendment of the 802.11 protocol suite is 802.11p, also known as Wireless Access in Vehicular Environments (WAVE). The protocol supports ITS and V2V/V2I communication [10]. The goal is to support communication between vehicles to improve road safety and traffic efficiency. The $802.11 \mathrm{p}$ protocol uses the $5.9 \mathrm{GHz}$ band and can offer data rates up to $27 \mathrm{Mbps}$ [11]. The estimated outdoor range is approximately 1000 meters [12].

\section{RELATED WORK}

Wireless short-range communication within quarries is a relatively unexplored area. Major vehicle manufacturers such as Volvo have products based mainly on cellular communication for maintenance and productivity monitoring. Few solutions on the market utilize low latency short-range communication for active safety applications and real-time productivity control in quarries. Hence, the available shortrange communication technologies and open spectrums have not been assessed or evaluated for this environment. In contrast, one can easily find published research for the general usage of wireless short-range communication in road vehicle environments.

Since ZigBee RF at $868 \mathrm{MHz}$ has a very low throughput and low energy consumption [13], it has mainly been used in local, low energy sensor networks. Very few have explored the range of this technology without energy consumption and throughput considerations in mind. We assume that this is due to the existence of few applications with these specific needs.

For 802.11 p networks, range has been explored for the ITS applications in VANETs. Huaqun et al. [14] investigate the number of received messages over the distance in meters in an 802.11p network. Their tests are conducted in an open area where the devices are moved apart up to a point where no more messages can be received. The communication range reaches up to 850 meters while dropping only two messages during a $1400 \mu$ interval time. At 900 meters, the device could no longer receive any messages.

Research shows that 802.11 p suffers from severe packet loss in conditions where several vehicles are within range and broadcasting at a high frequency [11]. This phenomenon is not expected within a normal quarry operation since the number of nodes within range will likely be significantly less than 100 . The speed within a quarry is relatively low compared to highways, so the required broadcast rate for safety messages should be less frequent.

Studies of range tests using $802.11 \mathrm{~g}$ can be found mainly for indoor WLAN environments. Very few evaluations assess quarry environments. The experimental study conducted by Wellens et al. [15] presents the relationship between goodput and distance of two nodes in an 802.11g network. The experiment takes place at a $2 \mathrm{~km}$ LOS highway using two laptops (one client and one server). The server laptop sits in the middle of the road while the client laptop passes by with high speed. The maximum distance achieved is approximately 750 meters while transmitting UDP packets of 750 bytes. When using a fixed rate of $11 \mathrm{Mbps}$, a range of 800 meters can be reached while sending UDP packets of 1250 bytes payload.

The community still lacks knowledge about the performance of wireless short-range communication standards within quarries. This paper presents a performance evaluation of three wireless short-range technologies at the open frequency spectrums $868 \mathrm{MHz}, 2.4 \mathrm{GHz}$, and $5.9 \mathrm{GHz}$ in a quarry environment. The evaluation utilizes the differences in allowed specified limits for transmit gain and available bandwidth [16]. The main evaluation criteria are made considering safety and fuel efficiency application usage. In this context, the paper derives its results from measurements in a real-world quarry environment.

\section{METHOD}

Short-range communication for VANET normally has relatively low latencies since it is not intended to use infrastructure access points that a cellular network or satellite communication depends on. Instead it uses direct communication between two communicating nodes. For safety messages, the available standards are not dependent on bandwidth and throughput. Messages are only a few bytes that must be shared several times per second. The key performance indicators (KPIs) for quarries used in our evaluation are communication range and PRR.

We made use of two test environments to evaluate the KPIs of the wireless technologies. A LOS study was conducted to evaluate the ideal performance of the test equipment. In addition, we performed two empirical studies at a quarry outside Gothenburg, Sweden. The LOS test took place at Volvo Cars Demo Center (VCDC) test track, which permits a $1.8 \mathrm{~km}$ straight LOS evaluation.

The first quarry measurement is taken at the top of the quarry, which allows us to evaluate communication at the highest levels of the site. The second quarry measurement is taken at the bottom level. We consider the latter more significant, as future quarry applications are intended for use in a pit in which articulated haulers and trucks operate. The testing equipment consists of two sets of hardware platforms. The first platform is made up of Alix boards with wireless interfaces for $802.11 \mathrm{p}$ and $802.11 \mathrm{~g}$ communication. For the 802.11 p setup we use a three meter coaxial cable to connect to the $6 \mathrm{dBi}$ ECOM6-5500 antenna, adding a cable loss of $1.7 \mathrm{~dB}$ per meter. The total output power is estimated at $33 \mathrm{dBm}$. The output power from the $802.11 \mathrm{~g}$ card is set to $20 \mathrm{dBm}$. The $5 \mathrm{dBi}$ ECOM5-2400 antenna gives a total output power of $20 \mathrm{dBm}$ total. Both $802.11 \mathrm{p}$ and $802.11 \mathrm{~g}$ transmit packets of 214 bytes using a fixed data rate of $6 \mathrm{Mbps}$.

The second platform contains a Waspmote board and gateway from Libelium. It also has a wireless interface for ZigBee RF communication from XBee and two $4.5 \mathrm{dBi}$ antennas. The XBee module S5 has a transmit power of $25 \mathrm{dBm}$. The coaxial cable causes a cable loss of $8.5 \mathrm{~dB}$. In total, we achieve an output power of $21 \mathrm{dBm}$ from the point of 
the $4.5 \mathrm{dBi}$ antenna. The low-rate communication protocol ZigBee RF transmits frames of 19, 20, and 21 bytes with a $20 \mathrm{Kbps}$ data rate. The variation in sent bytes results from a frame sequence, which functions as a counter to detect frame loss. It counts from 0 to 255 before it resets to zero and thus uses one, two, or three bytes in binary coded decimal format.

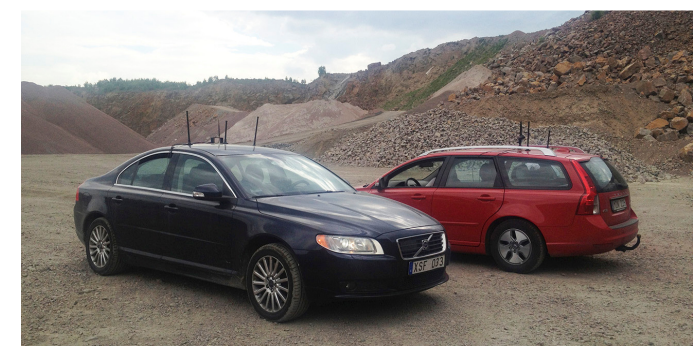

Figure 1. Cars used in the data collection with mounted roof antennas

We made use of two cars during the tests where one was responsible for transmitting while the other was set to receive packets. Figure 1 shows the Volvo S80 (receiving node) and the Volvo V50 (sending node) that were used during the measurements. Both vehicles were equipped with antennas, each responsible for providing communication for a certain technology. Each car also had a GPS module that allowed us to measure the distance between the communicating vehicles.

To facilitate the evaluation, all technologies are configured to transmit at a similar rate. Since the communication link must be robust, we experiment with how many packets per second (pps) we can transmit without decreasing the PRR. The Libelium equipment begins to perform unstably when transmitting at more than 4-5 pps. We therefore choose to set the transmission rate to approximately $2 \mathrm{pps}$, as this can be done without affecting communication quality.

The evaluation and data collection are done during normal Swedish daylight, sunny spring weather conditions without considerable moist, rain or snow. The experiments are repeated several times without noticing deviations in the results. The data presented are from one measurement since an aggregation would not be representative due to slight differences in the data collection, path and speed of the vehicle.

\section{RESULTS}

We discuss our results in the following three subsections according to the specified testing environment.

\section{A. LOS Range Measurements}

The $1.8 \mathrm{~km}$ VCDC LOS track is a road normally used for testing top speeds of new cars. We were able to use the facility while driving at the relatively low speed of $45 \mathrm{~km} / \mathrm{h}$, which was required for collecting detailed measurements (due to our equipment's low packet transmission rate).

Figure 2 shows the maximum distance in meters together with aggregated packet loss for each wireless protocol at the VCDC track. The maximum distance is the measured range at the time when we receive the last packet. The number of sent packets during the measurement is 376 for ZigBee RF, 462 for $802.11 \mathrm{~g}$ and 463 for $802.11 \mathrm{p}$.

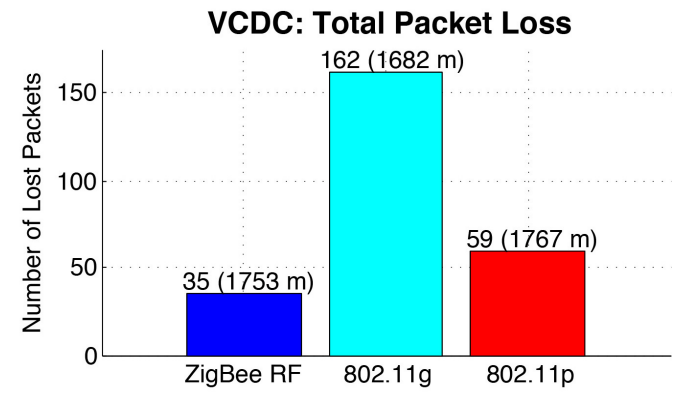

Figure 2. Packet loss at a point of maximum distance per technology

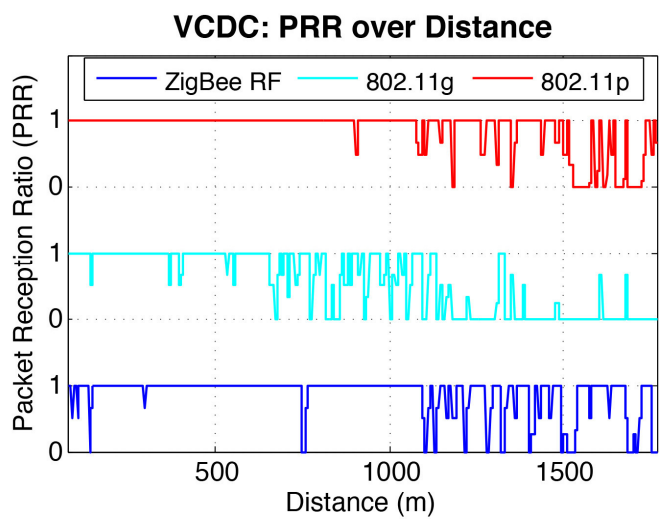

Figure 3. PRR over distance per technology at the VCDC track

Figure 3 displays the PRR over distance per wireless technology. The lines are stacked on top of each other where all standards have been given PRR values from 0 to 1 . A PRR value of 1 stands for full packet reception while 0 represents a state of $100 \%$ packet loss. The figure shows that $802.11 \mathrm{~g}$ performs well (with only minor connectivity issues) before its stability decreases just before 700 meters. After 1400 meters, $802.11 \mathrm{~g}$ experiences $100 \%$ packet loss, except at a few spots. The last $802.11 \mathrm{~g}$ packet is received at 1682 meters. In the meantime, ZigBee RF performs robustly except for some points where the connectivity was lost. At 1100 meters, ZigBee RF becomes less robust but can still frequently receive packets up till 1753 meters. 802.11p has almost 100\% PRR up to 1100 meters and still receives packets at distances up to 1767 meters, at which point we lose LOS. We are thus confident that both ZigBee RF and 802.11p could reach even further on a longer LOS track.

\section{B. Quarry Top Measurements}

The top of the quarry defines the outer area of the site and consists of a road surrounding the quarry. Few vehicles operate at this highest level where hills and rocks cause most NLOS problems. Figure 4 and Figure 5 show the PRR over distances for each standard at the quarry top. The different 802.11 technologies clearly experience simultaneous NLOS issues, but ZigBee RF seems unaffected by obstacles. 


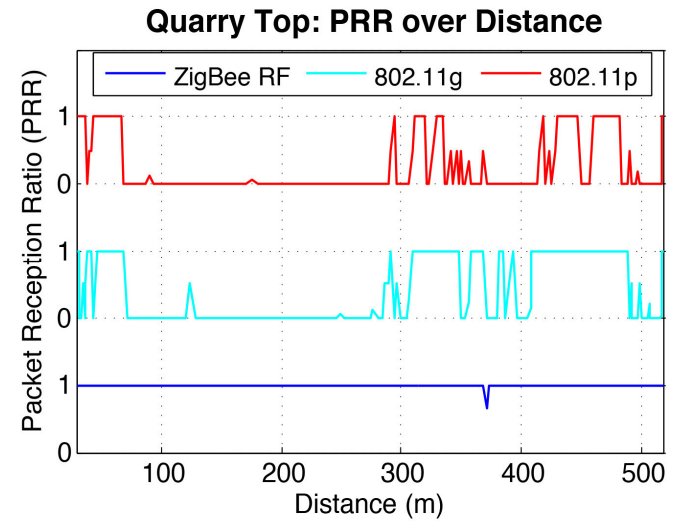

Figure 4. PRR over distance during the first 500 meters at the quarry top

Figure 4 illustrates the first half of our measurement, when the sending vehicle is driving away from the receiving vehicle. The figure shows that $802.11 \mathrm{~g}$ and $802.11 \mathrm{p}$ experience high packet loss at approximately 70 to 320 meters. On the other hand, both protocols can recover quickly after this point. $802.11 \mathrm{~g}$ provides almost full connectivity from 320 to 350 meters and again from 420 to 480 meters. ZigBee RF only loses one packet up to a distance of 520 meters.

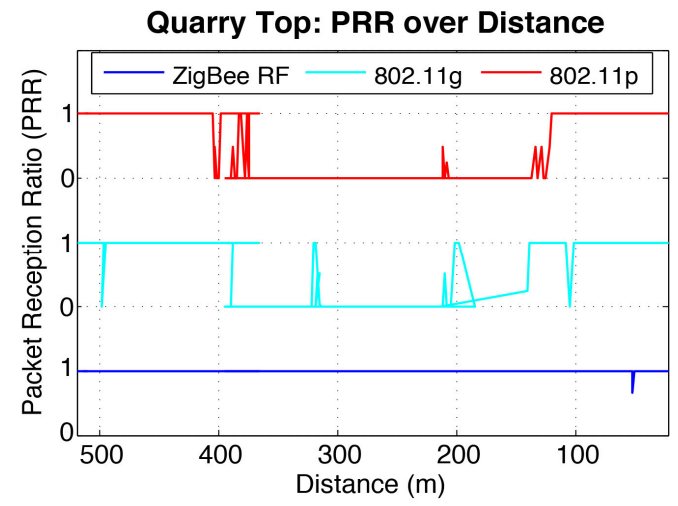

Figure 5. PRR over distance when driving back to the sending node

Figure 5 shows the second half of the test, in which the vehicle that receives packets drives back towards the vehicle that transmitted packets. The top quarry road contains NLOS spots, which can be seen between 410 and about 200 meters. $802.11 \mathrm{p}$ has communication difficulties at this distance, but it improves at 120 meters. $802.11 \mathrm{~g}$ recovers faster than $802.11 \mathrm{p}$ and is able to receive packets at times when the latter does not function at all. Again, ZigBee RF does not seem to be affected by NLOS. Figure 5 contains double lines at certain points for $802.11 \mathrm{~g}$ and $802.11 \mathrm{p}$ because the topology of the road caused the car to be at the same distance for multiple data samples.

Figure 6 shows the total number of lost packets for each technology after the quarry top test. During the measurement, ZigBee RF transmits 988 packets while $802.11 \mathrm{~g}$ and 802.11p send out 606 packets in total. ZigBee RF experiences the lowest packet loss, with only two lost packets during the whole test round (packet loss ratio of $0.2 \%$ ). $802.11 \mathrm{~g}$ performs second best at a loss of 164 packets $(27.1 \%)$.

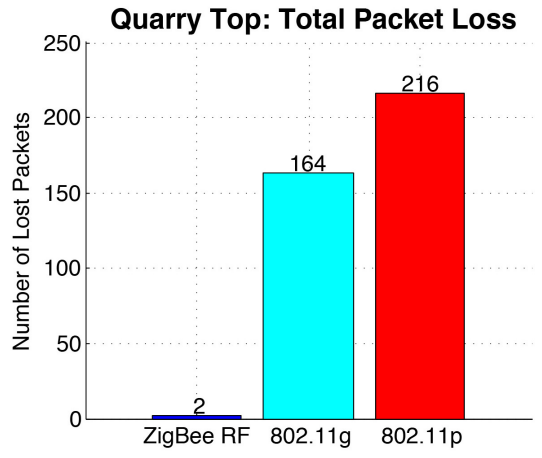

Figure 6. Total packet loss at the quarry top per wireless technology

802.11p did not perform as well as $802.11 \mathrm{~g}$ or ZigBee RF and experienced a total loss of 216 packets (35.6\%). By looking at the figures, it is evident that $802.11 \mathrm{p}$ on the $5.9 \mathrm{GHz}$ band was sensitive to rocks, hills, and other obstacles. ZigBee $\mathrm{RF}$ at $868 \mathrm{MHz}$ has the opposite behavior. It does not seem to be affected by NLOS, and it maintains a robust connection throughout the entire test round.

The Figures 4, 5, and 6 give a coherent image of the results from the top of the quarry. $802.11 \mathrm{p}$ is pending frequently between $100 \%$ and $0 \%$ PRR and experiences high packet loss. $802.11 \mathrm{~g}$ is also unstable at times, but it only loses 164 packets in total. ZigBee RF gives a robust performance and loses only two packets.

\section{Quarry Pit Measurements}

The quarry pit is the lowest part of the site where most of the work takes place. It is here where you will find typical quarry elements such as trucks, crushers and articulated haulers that produce and distribute sand and gravel. As a result of the production, piles of these materials are spread around the pit area. These are the main obstacles for wireless communication.

Figure 7 shows the total packet loss from the quarry pit test, compared to the 715 packets sent by ZigBee RF and 878 sent by $802.11 \mathrm{p}$ and $802.11 \mathrm{~g}$ respectively. Again we see that ZigBee RF experiences the lowest packet loss rate. Similar to the top quarry test, it only loses two packets in total $(0.3 \%)$. The second best performing technology this time is $802.11 \mathrm{p}$ with a loss of 283 packets (32.2\%). $802.11 \mathrm{~g}$ gives the least satisfying results as it loses 650 packets (74\%). During the quarry pit tests, we are able to see when the technologies experience packet loss due to obstacles from a graphical interface in the car. These mostly consist of piles of sand or stone. This time, it is $802.11 \mathrm{~g}$ that experiences most difficulties in handling the obstacles.

Figure 8 and 9 depict PRR over distance in the quarry pit. Figure 8 shows when we are driving away from the transmitting vehicle up to a distance of 400 meters before reaching the other end of the quarry. Figure 9 depicts the opposite scenario when we drive back towards the transmitting vehicle that is parked close to the mountain wall surrounding the pit. From Figures 8 and 9 it is evident that ZigBee RF is yet again the technology that can provide the most stable communication. During the first 400 meters, it has a constant 
PRR value of one. $802.11 \mathrm{~g}$, on the other hand, is unstable already from the start. Both figures show how it often has a PRR value of zero and only provides connectivity during the first and last 150 meters.

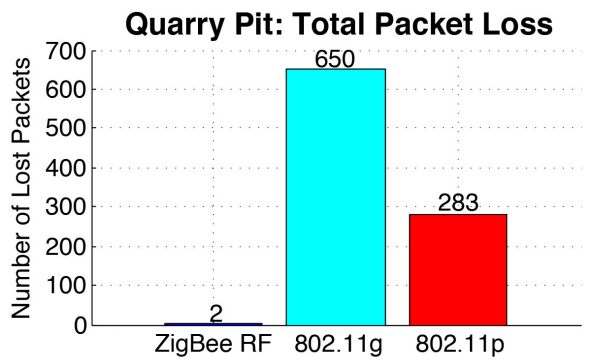

Figure 7. Quarry pit test showing packet loss for all technologies

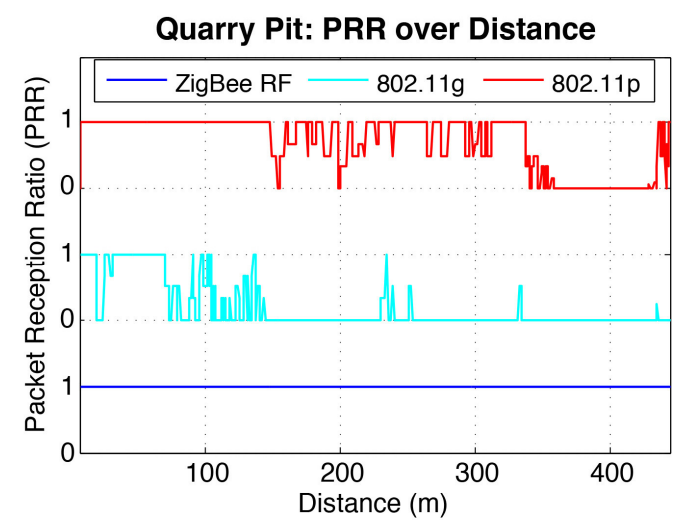

Figure 8. PRR over distance during the first 400 meters in the pit

802.11 p gives satisfyingresults in the pit. It has a $100 \%$ reception rate up to 150 meters from the sending node and can generally provide a stable connection. We see that $802.11 \mathrm{~g}$ starts to experience packet loss after 75 meters in Figure 8. This is caused by driving behind a large pile of sand. After this point, we do not have a clear LOS between the transmitting and receiving vehicle, and $802.11 \mathrm{p}$ also starts to lose packets. ZigBee RF operating on the low $868 \mathrm{MHz}$ band is able to penetrate the obstacles and deliver a $100 \%$ reception rate.

In Figure 9, it is possible to see when we are driving down in the lowest part of the pit with enclosing obstacles that blocks the LOS completely. This leads to a PRR value of 0 for all technologies, except ZigBee RF, somewhere between 380 and 170 meters. The only point when ZigBee RF loses any packets is after the NLOS spot where piles of stone are present that it cannot penetrate. $802.11 \mathrm{p}$ is able to provide a very robust connection after its recovery until the end of the route. In contrast, $802.11 \mathrm{~g}$ is not able to recover after the NLOS area and gives an overall weak impression.

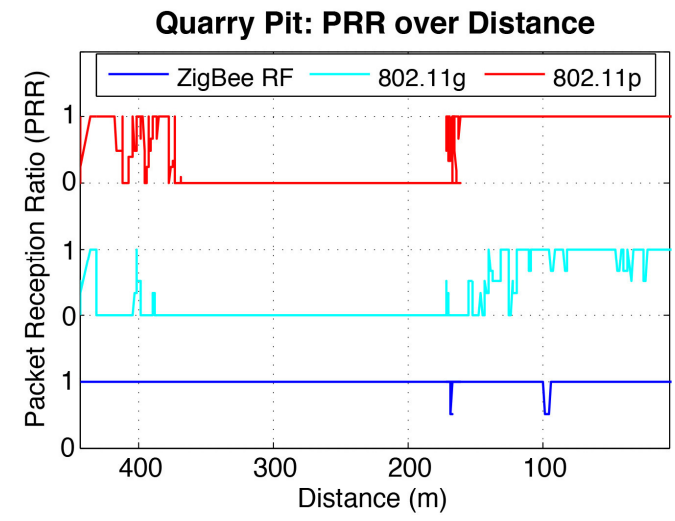

Figure 9. PRR in the pit when driving back towards the transmitting car

\section{CONCLUSIONS}

The measurements conducted during this work shows that two vehicles can communicate from much greater distances than initially expected. $802.11 \mathrm{p}$ is able to reach a range of 1767 meters in LOS conditions. We had estimated far shorter distances as the literature reports a maximum range of 1000 meters. In the quarry pit, $802.11 \mathrm{p}$ benefits from the reflecting surface of the enclosing mountain and provides a robust communication with low packet loss. The protocol is also able to recover fast once it leaves the NLOS spots. At the quarry top on the other hand, 802.11p gives one of the least satisfying results with a low PRR and a weak communication.

802.11g performs well at the quarry top but gives a poor result in the quarry pit where it loses the most packets out of all standards. 802.11g delivers unstable communication in the pit and is sensitive to NLOS areas and obstacles where it is unable to benefit from reflecting surfaces. The technology can nevertheless obtain an excellent LOS range result of 1682 meters. As reported by IEEE, only 150 meters are expected in an outdoor environment. Since $802.11 \mathrm{~g}$ performs poorly in the pit, we do not consider the protocol a suitable candidate for quarry applications.

Of all standards, ZigBee RF is the technology that provides the best results and seems unaffected by NLOS. It only loses two packets at the quarry top and pit where it delivers a stable communication link. It also achieves the second best range result of 1753 meters. The reported top range for ZigBee RF of $12 \mathrm{~km}$ in a clear LOS cannot be tested. However, we do see that the protocol can reach near the end of the $1.8 \mathrm{~km} \mathrm{VCDC}$ track. We therefore believe that ZigBee RF can reach further distances if tested at a longer LOS track. Following our results, the two best performing technologies for the quarry are 802.11p and ZigBee RF. These protocols are the opposite of each other in terms of frequency band; one is communicating in the $868 \mathrm{MHz}$ ISM band while the other at $5.9 \mathrm{GHz}$. This fact, however, can be seen as the key to their success in the quarry. The technology is either penetrating the pit obstacles using a low frequency or reflecting from the surface of the quarry mountain using a high frequency. Both strategies achieve similar results where the connectivity is maintained. From this perspective, the significant factor is the frequency rather than a specific protocol. Bandwidth has not been evaluated but can be foreseen as an important factor since there 
is a major difference between the allocations. Based on the communication needs, bandwidth may affect the preferred technology to use.

Output power is another relevant factor. In this context, it is important to clarify that $802.11 \mathrm{p}$ has an output power of $33 \mathrm{dBm}$, while the other technologies use 20 and $21 \mathrm{dBm}$. It is nevertheless not evident which protocol would be best in practice to optimize both fuel consumption and increase quarry safety. The problem with ZigBee RF is its low data rate of $20 \mathrm{Kbps}$ or less, which may be insufficient for future quarry applications, especially for safety applications that rely on low latencies. $802.11 \mathrm{p}$ is a promising technology for the quarry with data rates up to $54 \mathrm{Mbps}$ and a reasonable coverage range. It can, however, not provide as good coverage as ZigBee RF at $868 \mathrm{MHz}$. For this reason, we believe that a combination of both technologies would be the best choice for the quarry to simultaneously maximize range and throughput.

\section{FUTURE WORK}

The approach presented should be further evaluated at different quarries and for a longer duration. An extended study using actual quarry machines onsite in daily operation can better assess the actual communication requirements by taking into account distances between the machines and true operational interactions.

Extended measurements for throughput, message delays, and signal strength could be evaluated by stressing the technologies using different data rates, which should affect the communication quality. Moreover, this paper does not consider weather constraints; a study during rain, fog, and snow in a quarry that is open all year round may provide different results.

Finally there are more communication standards (e.g., 802.11n and 802.11ac) and frequency bands available in different regions/countries. The use of other communication systems and suppliers would complement the results presented in this study.

\section{ACKNOWLEDGMENT}

The authors want to acknowledge the valuable support in the setup and performance of the trials from Mr. Jakob Fryk and Mr. Edvin Valtersson at Volvo Global Trucks Technology. Furthermore, this research is supported by the Knowledge Foundation (KKS) through ITS-EASY, an Industrial Research School in Embedded Software and Systems, affiliated with Mälardalen University, Sweden. Chalmers Prof. Sally A. McKee helped polish our final presentation.

\section{REFERENCES}

[1] H. Hartenstein and K. Laberteaux, VANET Vehicular Applications and Inter-Networking Technologies, 2010.

[2] UEPG. (2012). European Aggregates Association. Available: http://www.uepg.eu/

[3] D. Rylander and J. Axelsson, "LEAN METHOD TO IDENTIFY IMPROVEMENTS FOR OPERATION CONTROL AT QUARRY SITES," presented at the ISARC Conference, Montreal, 2013.
[4] B. S. Dhillon, "Mining equipment safety: a review, analysis methods and improvement strategies," International Journal of Mining, Reclamation and Environment, vol. 23, pp. 168-179, 2009/09/01 2009.

[5] P. Baronti, P. Pillai, V. W. Chook, S. Chessa, A. Gotta, and Y. F. Hu, "Wireless sensor networks: A survey on the state of the art and the 802.15. 4 and ZigBee standards," Computer communications, vol. 30, pp. 1655-1695, 2007.

[6] L. Pengfei, L. Jiakun, N. Luhua, and W. Bo, "Research and application of zigbee protocol stack," in Measuring Technology and Mechatronics Automation (ICMTMA), 2010 International Conference on, 2010, pp. 1031-1034.

[7] Libelium. (2013). Waspmote Technical Guide. Available: http://www.libelium.com/uploads/2013/02/waspmote-technical_ guide_eng.pdf

[8] S. William. (2004) IEEE 802.11:Wireless LANs from a to n. 32-37. Available: http://doi.ieeecomputersociety.org/10.1109/MITP.2004.62

[9] A. B. Fadhah, A. Al-Lawati, S. Al-Maskari, A. Touzene, and A. AlKindi, "Experimental performance evaluation of Wireless $802.11 \mathrm{~b}$ networks," in Applications of Digital Information and Web Technologies, 2008. ICADIWT 2008. First International Conference on the, 2008, pp. 151-155.

[10] S. Eichler, "Performance evaluation of the IEEE 802.11 p WAVE communication standard," in Vehicular Technology Conference, 2007. VTC-2007 Fall. 2007 IEEE 66th, 2007, pp. 2199-2203.

[11] K. Sjöberg, Medium access control for vehicular ad hoc networks: Chalmers University of Technology, 2013.

[12] H. Guo, S. T. Goh, N. C. Foo, Q. Zhang, and W.-C. Wong, "Performance evaluation of $802.11 \mathrm{p}$ device for secure vehicular communication," in Wireless Communications and Mobile Computing Conference (IWCMC), 2011 7th International, 2011, pp. 1170-1175.

[13] J.-S. Lee, Y.-W. Su, and C.-C. Shen, "A comparative study of wireless protocols: Bluetooth, UWB, ZigBee, and Wi-Fi," in Industrial Electronics Society, 2007. IECON 2007. 33rd Annual Conference of the IEEE, 2007, pp. 46-51.

[14] G. Huaqun, G. Shen Tat, N. C. S. Foo, Z. Qian, and W. Wai-Choong, "Performance evaluation of $802.11 \mathrm{p}$ device for secure vehicular communication," in Wireless Communications and Mobile Computing Conference (IWCMC), 2011 7th International, 2011, pp. 1170-1175.

[15] M. Wellens, B. Westphal, and P. Mahonen, "Performance evaluation of IEEE 802.11-based WLANs in vehicular scenarios," in Vehicular Technology Conference, 2007. VTC2007-Spring. IEEE 65th, 2007, pp. 1167-1171

[16] Post och telestyrelsen. (2013, September). The Swedish Post and Telecom Authority (PTS). Available: http://www.pts.se/ 